\title{
PROBIOTIC LACTOBACILLUS REUTERI EFFECT'S ON THE LEVELS OF INTERLEUKIN-4 IN PERIODONTITIS PATIENTS AFTER SCALING AND ROOT PLANING
}

\author{
RIKA TANIA NARULITA SINULINGGA, YUNIARTI SOEROSO*, ROBERT LESSANG, \\ DEWI FATMA SUNIARTI SASTRADIPURA
}

Department of Periodontology, Faculty of Dentistry, Universitas Indonesia, Jakarta, Indonesia Department of Oral Biology, Faculty of Dentistry, Universitas Indonesia, Jakarta, Indonesia. E-mail: yuniarti_22@yahoo.co.id

Received: 01 August 2019, Revised and Accepted: 19 February 2020

\section{ABSTRACT}

Objective: Periodontal disease is an inflammatory disease principally caused by a complex interaction between pathogenic bacteria and immune responses. Probiotics stimulate the immune system of the oral mucosa by increasing the production of anti-inflammatory cytokines. Interleukin-4 (IL-4) is the anti-inflammatory cytokine that is most closely associated with the pathogenesis of periodontitis. This study assessed the effect of Lactobacillus reuteri (L. reuteri) containing probiotic lozenges toward clinical attachment loss (CAL) and IL-4 levels in periodontitis patients after scaling and root planing.

Materials and Methods: Clinical samples were collected from the gingival crevicular fluid of 16 periodontitis patients with pocket depth (PD) of $6 \mathrm{~mm}$. The samples were divided into two groups. The non-probiotic group included periodontitis patients treated through scaling and root planing (SRP) only $(n=8)$, whereas the probiotic group included periodontitis patients treated through SRP and probiotics $(n=8)$. The measurements of the clinical parameters of PD and CAL were included as diagnostic criteria. Probiotic lozenges were given daily for 14 days. IL-4 levels were measured in both groups using the enzyme-linked immunosorbent assay (ELISA) method.

Results: IL-4 levels decreased in the non-probiotic group and increased in the probiotic group, but there was no statistical difference between the groups. CAL decreased in both groups and was significantly different.

Conclusion: SRP with the consumption of probiotic-containing L.reuteri in periodontitis patients resulted in decreased CAL and increased IL-4 levels compared with SRP only.

Keywords: Probiotic, Interleukin-4 (IL-4), Periodontitis, Scaling and root planing.

(c) 2020 The Authors. Published by Innovare Academic Sciences Pvt Ltd. This is an open access article under the CC BY license (http://creativecommons. org/licenses/by/4. 0/) DOI: http://dx.doi.org/10.22159/ijap.2020.v12s2.PP-20

\section{INTRODUCTION}

Periodontal disease is an inflammatory disease of the periodontal tissue. It can destroy the periodontal ligament and alveolar bone form periodontal pockets, gingival recession, or combination [1]. Groups of specific microorganisms mainly cause periodontal diseases. An imbalance between pathogenic bacteria and immune responses can destroy the periodontal tissue [2]. Host immune response is a determining factor in the development of periodontal disease [3]. Periodontal treatment primarily includes scaling and root planing (SRP). However, in some cases, SRP is insufficient to treat periodontal disease. In the past 20 years, antibiotics have been administered as additional therapy [4]. Incorrect use of antibiotics can cause bacterial resistance and recolonization in the same site; therefore, a novel adjunct therapy needed for the periodontal disease [3].

Probiotics are living microorganisms, which can have beneficial health effects on the host [5]. Probiotics significantly influence the periodontium regarding plaque modification, halitosis management, changing anaerobic bacterial colonization, reducing pocket depth (PD), and clinical attachment loss (CAL)-associated with host cell responses [5]. Probiotics are broadly categorized into two genera: Lactobacillus and Bifidobacterium [5]. Penala et al. stated that the administration of Lactobacillus reuteri (L. reuteri) once daily for 14 days can be therapy for SRP and very effective in reducing periodontal pocket depth (PD) [6]. A study by Waerhug et al. stated that healing after occurs within 2 weeks, whereby the epithelial proliferation process stops within 7-14 days after SRP [7].

Periodontal disease is often associated with excessive production of pro-inflammatory cytokines or an imbalance between the production of pro-inflammatory and anti-inflammatory cytokines [8]. Anti-inflammatory cytokinesarea series ofimmunoregulatorymolecules that control the cytokine pro-inflammatory response [8]. The main anti-inflammatory cytokines are interleukin (IL)-4, IL-10, IL-12, IL-13, and IL-18 [8]. Probiotics stimulate and modulate the immune system of the oral mucosa by reducing pro-inflammatory cytokine production through action on the NFkB pathway and increasing antiinflammatory cytokines [9]. Among the inflammatory factors, IL-4 is the anti-inflammatory cytokine most closely associated with the pathogenesis of periodontitis through increasing Th2 proliferation, suppressing Th1 proliferation, and suppressing the Th1-mediated immune response [10].

Indonesia, there are many circulating probiotics in the form of tablets, lozenges, chewing gum, and toothpaste, but few studies on the effectiveness of these probiotics. A study has reported that probiotics containing L. reuteri can increase the secretion of anti-inflammatory cytokines such as IL-10 and IL-4 [11], whereas other studies have suggested that such probiotics are not effective against the increase in IL-4 [12]. This study aims to assess the effect of $L$.reuteri containing probiotic lozenges on CAL and IL-4 levels in periodontitis patients after SRP. We hypothesized that IL-4 levels would increase and CAL would decrease after SRP on consuming probiotics.

\section{MATERIALS AND METHODS}

Materials

Clinical samples were collected from patients at the Dental Teaching Hospital, Faculty of Dentistry, Universitas Indonesia, and laboratory 
work was conducted in the Laboratory of IMERI at the Faculty of Medicine, Universitas Indonesia.

This work was based on an observational study design. Clinical samples were collected from the gingival crevicular fluid (GCF) of 16 individuals (aged 20-56 years) between May and June 2019. All subjects gave written informed consent to participate in this study. Complete anamnesis was done by asking the chief complaint, medical history, allergies, systemic conditions, medications, smoking habits, and tooth brushing habits.

Detailed information was obtained by checking their oral cavity, measuring their oral hygiene index, PD, recession, and CAL. Inclusion criteria were subjects with periodontitis stages II, III, and IV, according to the World Workshop on the Classification of Periodontal and PeriImplant Diseases and Conditions 2017 [13]. Clinical parameters included pocket depth (PD) (=6 mm), PBI, and CAL ( $\geq 1 \mathrm{~mm})$. Exclusion criterias were: A history of systemic disease, bruxism or smoking, intake of antibiotics within the previous 3 months, pregnancy or breastfeeding, and scaling or other periodontal treatment $\leq 6$ months.

\section{Methods}

Clinical samples in the non-probiotic dan the probiotic groups were determined randomly. Periodontal clinical examination for PD was recorded using the Periodontal Probe UNC 15 (Osung Mnd, Korea). After the examination, a sample of GCF was collected from each individual before SRP. GCF was obtained from patients in both groups that had deep pockets $(\mathrm{PD}=6 \mathrm{~mm})$ in randomly selected sites of the healthy periodontal sulcus $(0-3 \mathrm{~mm})$. No probiotics were given in the non-probiotic group, whereas the probiotic group was given probiotics lozenges containing L. reuteri DSM 17938 daily for 14 d, which were marketed in Indonesia. Probiotic were given blindly. After 14 days, GCF was collected again from each individual in both groups. GCF was extracted to measure IL-4 levels using the ELISA Kit (Elabscience).

This study was approved by the Ethical Committee of Dental Research (KEPKG), Faculty of Dentistry, Universitas Indonesia (protocol number: 090430419).

Wilcoxon test was used for comparing IL-4 levels in the non-probiotic and the probiotic groups before and after SRP. Wilcoxon test was also used to conduct statistical analysis of CAL in the non-probiotic and the probiotic group. Data were analyzed using Statistical Package for the Social Sciences 23.0.

\section{RESULTS AND DISCUSSION}

Fig. 1 shows the average of IL-4 levels increased in the probiotic group but decreased in the non-probiotic group and Fig. 2 shows the average of CAL decreased in both groups.

The results of statistical analysis of IL-4 levels in both groups with abnormal data distribution using the Wilcoxon test method were not significantly different $\left(p_{\text {non-probiotic }}=0.17\right.$ and $\left.p_{\text {probiotic }}=0.139\right)$. CAL was significantly different in non-probiotic and probiotic groups $\left(p_{\text {non-probiotic }}=0.004\right.$ and $\left.p_{\text {probiotic }}=0.004\right)($ Table 1$)$.

The host immune response has an important role in damaging connective tissue and bone [14]. Probiotics increase the host immune response [15]. IL-4, a potent down regulator of macrophage function, can inhibit the secretion of pro-inflammatory cytokines such as IL-1, IL-6, and TNF- $\alpha$ to prevent periodontitis [15].

This study found that IL-4 levels in periodontitis patients increased after 14 days of SRP with probiotics, and IL-4 levels in periodontitis patients decreased after 14 days of SRP only. The decrease in IL-4 levels is caused by incomplete wound healing. The wound healing process is divided into phases: The inflammatory phase, the proliferation phase, and the maturation and remodeling phase [15]. The proliferation phase consists of granulation tissue formation, reepithelization, and neovascularization. Reepithelization occurs on the $14^{\text {th }}$ day, and IL-4 levels continue to decline until the $19^{\text {th }}$ day [16]. This is inconsistent with the results of the research by Warung et al. and Penala et al., which states that there is a healing of the pocket within 14 days. Increased levels of IL-4 in periodontitis patients who received SRP with probiotics showed that the probiotic L. reuteri accelerates the healing process [17]. This is consistent with the results of a study by Poutahidis et al., which states that probiotics increase the production of oxytocin which can accelerate the healing process [17].

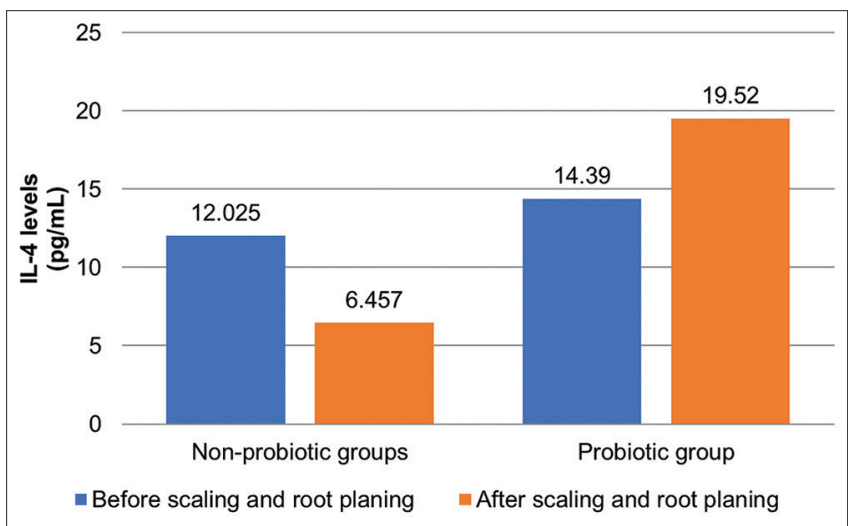

Fig. 1: The Average of IL-4 levels in the Non-probiotic and the probiotic groups

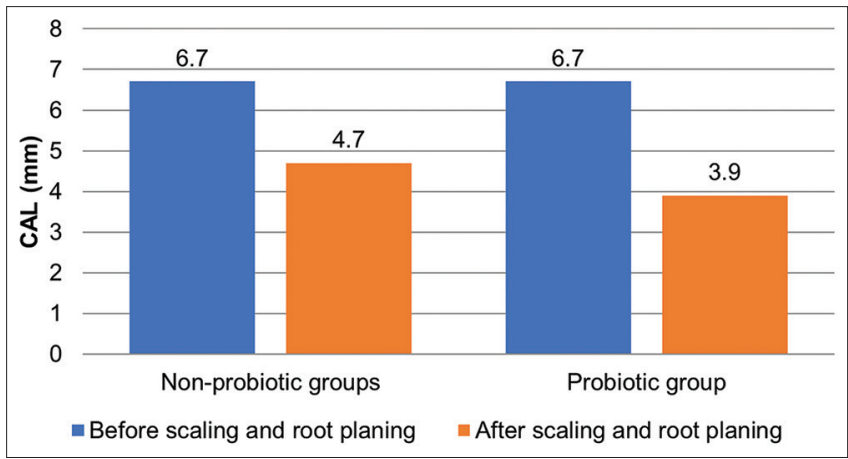

Fig. 2: The average of CAL in the Non-probiotic and the probiotic groups

Table 1: CAL and IL-4 levels in the non-probiotic and the probiotic groups`

\begin{tabular}{|c|c|c|c|c|c|c|}
\hline & & \multicolumn{2}{|l|}{ Before SRP } & \multicolumn{2}{|l|}{ After SRP } & \multirow[t]{2}{*}{$\mathbf{p}$} \\
\hline & & Mean \pm SD & Med (Min-Max) & Mean \pm SD & Med (Min-Max) & \\
\hline \multirow[t]{2}{*}{ Non-probiotic } & $\mathrm{CAL}(\mathrm{mm})$ & $6.70 \pm 0.82$ & $6.50(6.00-8.00)$ & $4.70 \pm 0.67$ & $5.00(4.00-6.00)$ & $0.004^{*}$ \\
\hline & IL-4 level (pg/mL) & $12.02 \pm 4.42$ & $11.70(5.68-18.79)$ & $6.45 \pm 2.56$ & $5.68(4.34-13.24)$ & 0.17 \\
\hline \multirow[t]{2}{*}{ Probiotic } & $\mathrm{CAL}(\mathrm{mm})$ & $6.70 \pm 0.82$ & $6.50(6.00-8.00)$ & $3.90 \pm 1.37$ & $4.00(2.00-6.00)$ & $0.004^{*}$ \\
\hline & IL-4 level (pg/mL) & $14.39 \pm 11.42$ & $10.60(4.1-39.85)$ & $19.52 \pm 8.69$ & $20.23(7.96-35.23)$ & 0.139 \\
\hline
\end{tabular}

SD: Standard deviation; *Significant difference (Wilcoxon test, $\mathrm{p}<0.05$ ) 
Probiotics can also prevent periodontal disease [18]. Periodontal pocket shallowing in periodontitis patients may happen as a result of SRP and the administration of probiotics. Periodontal pocket shallowing can cause a reduction in CAL [18]. SRP can eliminate bacteria as the main cause of periodontitis. Lactic acid produced from probiotics can penetrate the bacterial membrane and acidify the cytoplasm by inhibiting the proliferation of periodontal disease-causing bacteria such as Porphyromonas gingivalis and Prevotella intermedia [18]. Reuterin, a bacteriocin produced by $L$.reuteri, has antibacterial activity against Gram-negative bacteria that cause periodontal disease [18].

IL-4 levels in the non-probiotic and the probiotic group before and after SRP were not significantly different. It should also be considered that saliva composition, including its inflammatory mediators' content, naturally varies in different individuals and is affected by several factors and conditions. As for this study, the inconclusive results reported could be a result of the wide range of the subjects' age, poor compliance of the subjects in the treatment group regarding probiotic consumption, and the relatively short sampling time (14 days) after treatment in both groups.

\section{CONCLUSION}

SRP is the main therapy for periodontitis. This study revealed that consumption of L. reuteri-containing probiotic lozenges alongside SRP in periodontitis patients resulted in decreased CAL and increased IL-4 levels compared with SRP only. Further studies are needed to address the changes in IL-4 levels in the course of periodontitis, and the effect of L. reuteri on oral health in humans.

\section{FUNDING}

This project was supported by a grant from HIBAH PITA UI 2019, Universitas Indonesia.

\section{AUTHORS' CONTRIBUTIONS}

All the authors have contributed equally

\section{CONFLICTS OF INTEREST}

Declared none.

\section{REFERENCES}

1. Saini R, Marawar PP, Shete S, Saini S. Periodontitis, a true infection. J Glob Infect Dis 2009;1:149-50.
2. Chickanna B, Prabhuji ML, Nagarjuna MS. Host-bacterial interplay in periodontal disease. J Int Clin Dent Res Organ 2015;7:44-50.

3. Gupta G. Probiotics and periodontal health. J Med Life 2011;4:387-94.

4. Soeroso Y, Akase T, Sunarto H, Kemal Y, Salim R, Octavia M. The risk reduction of recurrent periodontal pathogens of local application minocycline $\mathrm{HCl} 2 \%$ gel, used as an adjunct to scaling and root planing for chronic periodontitis treatment. Ther Clin Risk Manag 2017; 13:307-14.

5. Chatterjee A, Bhattacharya H, Kandwal A. Probiotics in periodontal health and disease. J Indian Soc Periodontol 2015;15:23-8.

6. Martin-Cabezas R, Davideau JL, Tenenbaum H, Huck O. Clinical efficacy of probiotics as an adjunctive therapy to non-surgical periodontal treatment of chronic periodontitis: A systematic review and meta-analysis. J Clin Periodontol 2016;43:520-30.

7. Ramseier CA, Rasperini G, Batia S, Giannobile WV.Advanced regenerative technologies for periodontal tissue repair. Periodontol 2000 2012;59:1-19.

8. Cetinkaya B, Guzeldemir E, Ogus E, Bulut S. Proinflammatory and anti-inflammatory cytokines in gingival crevicular fluid and serum of patients with rheumatoid arthritis and patients with chronic periodontitis. J Periodontol 2013;84:84-93.

9. Koduganti RR, Sandeep N, Guduguntla S, Gorthi VS. Probiotics and prebiotics in periodontal therapy. Indian J Dent Res 2011;22:324-30.

10. Jia XW, Yuan YD, Yao ZX, Wu CJ, Chen X, Chen XH, et al. Association between IL-4 and IL4R polymorphisms and periodontitis: A metaanalysis. Dis Markers 2017;2017:8021279.

11. Rocha-Ramirez LM, Pérez-Solano RA, Castañón-Alonso SL, Guerrero SS, Pacheco AR, Garibay MG, et al. Probiotic Lactobacillus strains stimulate the inflammatory response and activate human macrophages. J Immunol Res 2017;2017:4607491.

12. Twetman S, Derawi B, Keller M, Ekstrand K, Yucel-Lindberg T, Stecksen-Blicks C. Short-term effect of chewing gums containing probiotic Lactobacillus reuteri on the levels of inflammatory mediators in gingival crevicular fluid. Acta Odontol Scand 2009;67:19-24.

13. Caton JG, Armitage G, Berglundh T, Chapple ILC, Jepsen S, Kornman KS, et al. A new classification scheme for periodontal and peri-implant diseases and conditions introduction and key changes from the 1999 classifiaction. J Clin Periodontol 2018;45:S20.

14. Van Koppen CJ, Hartmann RW. Advances in the treatment of chronic wounds: A patent review. Expert Opin Ther Pat 2015;25:931-7.

15. Jain N, Joseph R, Balan S, Arun R, Banerjee M. Association of interleukin-4 and interleukin-17F polymorphisms in periodontitis in Dravidia ethnicity. Indian J Hum Genet 2013;19:58-64.

16. Michopoulou A, Rousselle P. How do epidermal matrix metalloproteinases support re-epithelialization during skin healing? Eur J Dermatol 2015;25 Suppl 1:33-42.

17. Levkovich T, Poutahidis T, Smilie C, Varian BJ, Ibrahim YZ, Lakritz JR, et al. Probiotic bacteria induce a "glow of health". PLoS One 2013;8:e53867.

18. Shetty A, Bhandary R, Thomas B, Ramesh A, Hedge P. Probiotic significance in periodontics a short review. J Indian Soc Periodontol 2017;2:57-60. 